\title{
木质素和氮含量对植物残体分解的影响
}

\author{
黄 耀 ${ }^{1,2}$ 沈 雨 ${ }^{1}$ 周 密 $^{1}$ 马瑞升 ${ }^{1}$ \\ (1 南京农业大学资源与环境科学学院, 南京 210095) \\ (2 中国科学院大气物理研究所大气边界层物理和大气化学国家重点实验室 北京 100029)
}

摘 要 在 $25{ }^{\circ} \mathrm{C}$ 和水分含量为 $400 \mathrm{~g} \mathrm{~kg}^{-1}$ ( 以风干土计) 条件下对 19 种植物残体进行培养实验, 同时进行田间填 埋试验，研究残体的木质素和 $\mathrm{N}$ 含量对其在土壤中分解的影响。相关分析表明，不同植物残体的分解速率与其初 始全 $\mathrm{N}$ 含量呈正相关，与初始木质素含量、木质素与 $\mathrm{N}$ 含量之比呈负相关。逐步回归分析进一步表明 植物残体的 $\mathrm{C}$ 分解与全 $\mathrm{N}$ 及木质素含量的数学关系可表达成: $Y=B_{0}+B_{1} N+B_{2} L$ 。式中, $B_{0} 、 B_{1}$ 和 $B_{2}$ 为回归系数, $N$ 和 $L$ 分 别表示植物残体的初始全 $\mathrm{N}$ 含量及木质素含量。 $Y$ 可分别表示为植物残体 $\mathrm{C}$ 分解的一级动力学常数、培养 9 周的 $\mathrm{CO}_{2}-\mathrm{C}$ 矿化率和填埋 23 周的重量损失率 相应的复相关系数 $\left(R^{2}\right)$ 分别为 $0.812^{* *} 、 0.828^{* *}$ 和 $0.799^{* *}$ 。此外, 实验 室培养测定的 $\mathrm{CO}_{2}-\mathrm{C}$ 矿化率和填埋试验的残体重量损失率之间具有良好的一致性 $\left(R^{2}=0.863^{* *}, n=17\right)$ 。

关键词 植物残体 分解 $\mathrm{N}$ 含量 木质素含量 定量关系

\section{DECOMPOSITION OF PLANT RESIDUE AS INFLUENCED BY ITS LIGNIN AND N ITROGEN}

\author{
HUANG $\mathrm{Yao}^{1}{ }^{2}$ SHEN Yu ${ }^{1}$ ZHOU $\mathrm{Mi}^{1}$ and MA Rui-Sheng ${ }^{1}$ \\ (1 College of Resources and Environmental Sciences , Nanjing Agricultural University , Nanjing 210095 , China) \\ (2 LAPC ,Institute of Atmospheric Physics, the Chinese Academy of Sciences , Beijing 100029, China)
}

\begin{abstract}
To quantitatively investigate the influence of plant nitrogen and lignin content on the residue carbon decomposition, $\mathrm{CO}_{2}$-C was measured from an incubation experiment with 19 plant residues plus soil under $25{ }^{\circ} \mathrm{C}$ and water content of $400 \mathrm{~g} \mathrm{~kg}^{-1}$ air dried soil over a 9-week period. The 19 residues were sampled from different organs with 13 plants, including Glycine max, Vigna sesquipedalis , Dioscorea esculenta , Luffa cylindrical , Oryza sativa , Zea mays , Triticum aestivum, Saccharum officinarum, Festuca arundinacea , Acorus calamus, Trapa quadrispinosa, Pittosporum tobira and Firmiana simplex. Weight loss of these residues was also obtained from a field burying experiment over a 23 -week period by using litter bag method.
\end{abstract}

Decomposition of plant residues during the early 3-week incubation period was fast with a wide variation. Thereafter the decomposition decreased and the variation among residues became gradually insignificant. $\mathrm{Cu}-$ mulative amount of $\mathrm{CO}_{2}-\mathrm{C}$ released over the 9-week incubation period was found to be different among the residues. Average of the cumulative $\mathrm{CO}_{2}-\mathrm{C}$ emission for all 19 residues was $178.0 \mathrm{mg}$, ranging from $103.6 \mathrm{mg}$ for Oryza sativa root to $256.2 \mathrm{mg}$ for Pittosporum tobira leaf. Correlation analysis indicated that decomposition of the plant residue was positively dependent on its initial nitrogen content and negatively dependent on its initial lignin content as well as the ratio of lignin to nitrogen. A further investigation suggested that the decomposition can be quantitatively described by $Y=B_{0}+B_{1} N+B_{2} L$. Parameters $B_{0}, B_{1}$ and $B_{2}$ are regression coefficients. The $N$ and $L$ represent the initial contents of nitrogen and lignin for a given residue, respectively. The $Y$ could be either the first-order decay rate $\left(B_{0}=3.51 \times 10^{-2}, B_{1}=4.61 \times 10^{-4}, B_{2}=-1.53 \times\right.$ $\left.10^{-4}, R_{2}=0.812^{* *}, n=19\right)$, or the percentage of $\mathrm{CO}_{2}$-C released over the 9-week period $\left(B_{0}=100, B_{1}\right.$ $\left.=0.974, B_{2}=-0.364, R^{2}=0.828^{* *}, n=19\right)$, or the percentage of weight loss over the 23-week period in the field burying experiment $\left(B_{0}=150, B_{1}=1.496, B_{2}=-0.572, R_{2}=0.799^{* *}, n=17\right)$. In addition , a good agreement $\left(R^{2}=0.863^{* *}, n=17\right)$ existed between amounts of the $\mathrm{CO}_{2}$-C released from the incubation and the weight loss from the field burying experiment.

It is concluded that decomposition of the plant residue either expressed as $\mathrm{CO}_{2}$ - $\mathrm{C}$ emission in the laboratory incubation or as weight loss in the field burying experiment was significantly dependent on its initial contents of nitrogen and lignin. Residues with higher nitrogen decomposed fast, while those with higher lignin content decomposed slowly. Difference in the decomposition for various plant residues can be well identified over a 9- 
week incubation under $25{ }^{\circ} \mathrm{C}$ and water content of $400 \mathrm{~g} \mathrm{~kg}^{-1}$ air dried soil. A further conclusion is that approximately $80 \%$ of the variability in the decomposition can be quantitatively expressed by a linear combination of the initial contents of nitrogen and lignin for a given residue. The result also suggests that fraction of the labile carbon for a given plant reside could be estimated from weight loss in the field burying experiment over a 23-week period.

Key words Plant residue , Decomposition , Nitrogen content , Lignin content , Quantitative relationship

气候变暖是当今全球性的环境问题,大气中 $\mathrm{CO}_{2}$ 浓度的不断增加对气候变暖起着极其重要的作 用。全球约有 $1.5 \times 10^{15} \mathrm{~kg}$ 碳以土壤有机质形式存 在，总量是大气中的两倍 (Eswaran et al. , 1993 ; Kirschbaum, 1995) ,分解产生的 $\mathrm{CO}_{2}$ 在全球 $\mathrm{CO}_{2}$ 排 放总量中占有相当比重 (Schimel，1995)。土壤中 $\mathrm{CO}_{2}$ 的排放主要来自土壤原有有机质和外源有机质 (如植物的凋落物、根茬及人为的有机物投入) 的分 解过程。许多研究表明, 大气中 $\mathrm{CO}_{2}$ 浓度的增加使 得植被的净初级生产力提高 (Weerakoon et al. , 1999 ;Morgan et al . , 2001) , 因而有更多的植物残体 进入土壤, 增加土壤 $\mathrm{C}$ 储量, 同时亦促进了土壤中 $\mathrm{CO}_{2}$ 的排放。因此, 研究植物残体在土壤中的分解 对于定量描述地-气系统 C 交换过程及其对气候变 化的影响具有重要意义。近年来, 国内外许多研究 集中在土壤类型(黄东迈等, 1998; Parshotam et al. , 2000)、水热条件 (Lloyd \& Taylor, 1994;Reichstein \& Bednorz , 2000 ;Chambers et al . , 2000)、 $\mathrm{CO}_{2}$ 浓度增加 (Sowerby et al ，2000) 和肥料施用 (Kwabiah et al. , 1999 ;Agren et al. , 2001) 等对植物残体分解的影
响,也有研究表明植物残体的分解速率与其木质素 和 $\mathrm{N}$ 含量有关(Rutigliano et al. , 1996)。然而, 关于 植物残体化学组分对其分解过程影响的定量研究则 不多见。本文试图通过对 19 种植物残体的实验室 培养测定及大田填埋实验结果, 阐明植物残体分解 与木质素及 $\mathrm{N}$ 含量间的数量关系。

\section{1 材料和方法}

\section{1 供试材料与测定项目}

19 种残体取自 13 种植物的不同器官，包括豆 科植物 (大豆 Glycine max , 豇豆 Vigna sesquipedalis)、 薯蓣科植物 ( 甘薯 Dioscorea esculenta) 、葫芦科植物 (丝瓜 Luffa cylindrica)、禾本科植物 (水稻Oryza sati$v a$,玉米 Zea mays , 小麦 Triticum aestivum, 甘蔗 Saccharum officinarum、高羊茅 Festuca arundinacea)、天南 星科植物 (菖蒲 Acorus calamus)、菱科植物 (菱角 Trapa quadrispinosa)、海桐科植物 (海桐 Pittosporum tobira)、梧桐科植物(法国梧桐 Firmiana simplex)。各 供试材料采回后洗净, $90{ }^{\circ} \mathrm{C}$ 杀青, $70{ }^{\circ} \mathrm{C}$ 烘干至恒重 备用。化学组分的测定包括总 $\mathrm{C}$ 、全 $\mathrm{N}$ 及木质素含 量。测定结果见表1, 可以看出, 各组分的测定值均

表 1 植物残体的化学性质

Table 1 Chemical properties of plant residue

\begin{tabular}{|c|c|c|c|c|c|}
\hline $\begin{array}{c}\text { 植物类型 } \\
\text { Family } \\
\end{array}$ & $\begin{array}{c}\text { 植物 } \\
\text { Species }\end{array}$ & $\begin{array}{c}\text { 残体 } \\
\text { Residue }\end{array}$ & $\begin{array}{c}\text { 总 C Total carbon } \\
\left(\mathrm{g} \cdot \mathrm{kg}^{-1}\right)\end{array}$ & $\begin{array}{c}\text { 全 N Total nitrogen } \\
\left(\mathrm{g} \cdot \mathrm{kg}^{-1}\right)\end{array}$ & $\begin{array}{c}\text { 木质素 Lignin } \\
\left(\mathrm{g} \cdot \mathrm{kg}^{-1}\right)\end{array}$ \\
\hline \multirow[t]{3}{*}{ 豆科 Fabacea } & 大豆 Glycine max & 叶片 Leaf & 437.4 & 35.2 & 245.8 \\
\hline & & 茎 Stalk & 417.6 & 22.1 & 195.4 \\
\hline & 豇豆 Vigna sesquipedalis & 茎 Stalk & 453.0 & 19.9 & 192.7 \\
\hline \multirow[t]{2}{*}{ 薯蓣科 Dioscoreaceae } & 甘薯 Dioscorea esculenta & 叶片 Leaf & 443.3 & 23.5 & 221.0 \\
\hline & & 茎 Stalk & 404.1 & 11.0 & 157.7 \\
\hline 葫芦科 Cucurbitaceae & 丝瓜 Luffa cylindrica & 叶片 Leaf & 353.1 & 30.9 & 193.8 \\
\hline \multirow{8}{*}{ 禾本科 Gramineae } & 玉米 Zea mays & 叶片 Leaf & 394.2 & 6.9 & 176.6 \\
\hline & & 茎 Stalk & 434.7 & 5.0 & 171.9 \\
\hline & & 根 Root & 398.9 & 5.3 & 195.4 \\
\hline & 水稻 Oryza sativa & 秸秆 Straw & 384.3 & 11.9 & 189.5 \\
\hline & & 根 Root & 459.5 & 9.5 & 214.0 \\
\hline & 小麦 Triticum aestivum & 根 Root & 441.2 & 8.7 & 232.8 \\
\hline & 甘蔗 Saccharum officinarum & 茎 Stalk & 472.0 & 4.0 & 201.1 \\
\hline & 高羊茅 Festuca arundinacea & 叶片 Leaf & 430.3 & 24.9 & 186.1 \\
\hline 天南星科 Araceae & 菖蒲 Acorus calamus & 叶片 Leaf & 449.5 & 21.4 & 220.0 \\
\hline 菱科 Trapaceae & 菱角 Trapa quadrispinosa & 茎 Stalk & 352.7 & 23.4 & 223.7 \\
\hline \multirow[t]{2}{*}{ 海桐科 Pittosporaceae } & 海桐 Pittosporum tobira & 叶片 Leaf & 431.8 & 15.4 & 173.1 \\
\hline & & 叶柄 Leafstalk & 426.5 & 7.4 & 182.4 \\
\hline 梧桐科 Sterculiaceae & 法国梧桐 Firmiana simplex & 叶片 Leaf & 483.9 & 17.8 & 254.0 \\
\hline
\end{tabular}


在一个较大的范围内变化, 说明供试材料具有较广 泛的代表性。

\section{2 实验室培养实验}

Vestergaard 等 (2001) 研究指出, 破碎植物残体并 不促进其分解。为了使残体样品具有均一性, 供试 材料经破碎, 选取 $10 \sim 20$ 目之间的样品 $1.0 \mathrm{~g}$ 与 $100 \mathrm{~g}$ 风干土样充分混合并加 $40 \mathrm{~g}$ 去离子水, 置于 $650 \mathrm{ml}$ 的呼吸瓶中在 $25{ }^{\circ} \mathrm{C}$ 下遮光密闭培养。供试 土壤选取有机 $\mathrm{C}$ 含量很低的薄层小粉白土, 总 $\mathrm{C}$ 和 全 $\mathrm{N}$ 含量分别为 $5.2 \mathrm{~g} \mathrm{~kg}^{-1}$ 和 $0.74 \mathrm{~g} \mathrm{~kg}^{-1}, \mathrm{pH}$ 值为 $7.4,<0.002 \mathrm{~mm}$ 粘粒含量为 $262 \mathrm{~g} \mathrm{~kg}^{-1}$ 。

呼吸瓶为相通的一大一小的锥形瓶, 大锥形瓶 口接有存放干燥剂 $\left(\mathrm{CaCl}_{2}\right)$ 的 $\mathrm{U}$ 型管, 主要用于在通 气时吸收空气中的水和 $\mathrm{CO}_{2}$, 同时瓶口用蜡密封。 小雉形瓶存放 $\mathrm{NaOH}$ 溶液, 每次用针筒注入标定溶 液 $10 \mathrm{ml}$,用橡胶塞封口，经培养一段时间后用针筒 将 $\mathrm{NaOH}$ 溶液取出, 根据 $\mathrm{NaOH}$ 溶液浓度的变化, 计 算出该培养时段内 $\mathrm{CO}_{2}$ - $\mathrm{C}$ 的释放量(周立祥 ,1995)。 每种植物残体设置两个重复, 另设空白对照。培养 过程中定期通气以补充氧气，第一周每天 1 次, 其后 2 到 3 天 1 次, 最后 3 周每 5 到 6 天 1 次,每次 30 min。以往的培养实验研究 (黄耀等, 2002) 表明, 不 同有机物料在培养前期 ( $0 \sim 30 \mathrm{~d}$ 左右) 的分解速率 最快, 此后便逐步下降, 在培养 $30 \mathrm{~d}$ 以后, 分解速率 变慢且随培养时间的变化幅度减小, 同时, 不同有机 物料间 C 分解速率的差异也不甚明显。因而本研 究设定的培养时间为 9 周。

\section{3 田间填埋试验}

不同植物残体田间分解率的确定采用网袋填埋 法(刘世梁等 2001) 填埋试验设在江苏省南京市江 宁县秼陵镇稻麦轮作区。试验区土壤的基本性状 为 $\mathrm{pH}$ 值 6.7、总 $\mathrm{C} 19.4 \mathrm{~g} \mathrm{~kg}^{-1}$ 、全 $\mathrm{N} 1.90 \mathrm{~g} \mathrm{~kg}^{-1}$ 、 $<0.002 \mathrm{~mm}$ 粘粒含量为 $490 \mathrm{~g} \mathrm{~kg}^{-1}$ 。各供试植物残 体剪断至 $3 \sim 5 \mathrm{~cm}$ 长, 称重 $10 \mathrm{~g}$ 装入 280 目尼龙网 袋中密封, 使其在分解过程中水分和气体可以自由 运动, 但未分解的有机物料却不能逸出, 而且土壤粘 粒亦不可侵入。采用完全随机分布方式，将装有不 同植物残体的网袋于 2000 年 12 月 21 日填埋于耕 作层, 各植物残体设置 3 个重复。2 3 周后将样品取 出, 洗去外部泥土, $70{ }^{\circ} \mathrm{C}$ 烘干至恒重, 并称重, 然后 计算出植物残体的重量损失。填埋期土壤平均温度 为 $12.2{ }^{\circ} \mathrm{C}$, 平均湿度为 $95 \%$ WFPS( Water filled pore space)。

\section{2 结果与讨论}

\section{1 培养实验}

用常规的差减法, 即用土壤加植物残体培养测 定的 C 释放量减去对照土壤的 C 释放量, 其差值即 为残体的 C 分解量。在培养前期 (3 周左右), 有机 $\mathrm{C}$ 的分解速率较快，此后便逐步减慢且随培养时间 的变化幅度减小。同时，不同残体间 $\mathrm{C}$ 分解速率的 差异也不甚明显 (图 1)。Murayama(1984)认为, 植物 残体的有机 C 可分为两个组分, 即易分解组分 (如 糖类、淀粉等)与难分解组分 (如木质素等)。易分解 的有机 C 在第一阶段得以快速分解, 而难分解组分 则分解比较缓慢。在 9 周的培养期内，不同植物残 体的 $\mathrm{CO}_{2}$ - $\mathrm{C}$ 累积分解量具有明显的差异 (表 2),19 种植物残体的平均 $\mathrm{CO}_{2}-\mathrm{C}$ 累积分解量为 $178 \mathrm{mg}$, 海 桐叶最高 $(256.2 \mathrm{mg})$, 水稻根最低 $(103.6 \mathrm{mg})$ 。

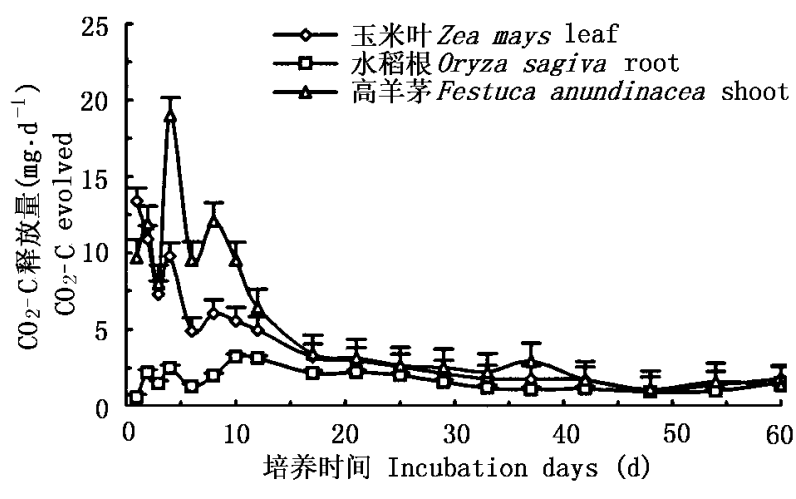

图 1 不同植物残体有机 C 分解速率

Fig.1 Decomposition rate of carbon from different plant residues

根据培养 9 周共 18 次的测定结果 (表 2)，各植 物残体的 $\mathrm{CO}_{2}-\mathrm{C}$ 释放量用以下一级动力学方程进行 模拟 :

$$
C=C_{0}[1-\exp (-k t)]
$$

式中, $C_{0}$ 和 $C$ 分别表示植物残体的初始含 $\mathrm{C}$ 量 $(\mathrm{mg})$ 和培养时间为 $t(\mathrm{~d})$ 时的累积 $\mathrm{CO}_{2}$-C 释放量 $(\mathrm{mg}), k$ 为一级动力学常数 $\left(\mathrm{d}^{-1}\right)$ 。由表 3 可以看 出, 不同植物残体的分解速率常数差异十分显著。 丝瓜叶的分解速率常数 $(k)$ 为 $2.28 \times 10^{-2}\left(\mathrm{~d}^{-1}\right)$, 而 水稻根的分解速率常数为 $0.44 \times 10^{-2}\left(\mathrm{~d}^{-1}\right)$, 两者相 差一个数量级。经过 9 周的培养, 前者的 $\mathrm{CO}_{2}-\mathrm{C}$ 矿 化率 (累积 $\mathrm{CO}_{2}-\mathrm{C}$ 释放量占残体含 $\mathrm{C}$ 量的百分比) 为 $59.7 \%$, 而后者仅为 $22.5 \%$ 。表 2 和表 3 的结果同 时表明 就总体趋势而言, 不同植物科之间的 $\mathrm{CO}_{2}-\mathrm{C}$ 矿化无明显差异, 但不同植物器官间差异明显, 叶片 分解最快, 茎次之, 根最慢。

根据表 1 和表 3 的结果, 分别将不同植物残体 
表 2 不同植物残体的 $\mathrm{CO}_{2}-\mathrm{C}$ 累积分解量 $(\mathrm{mg})$

Table 2 Cumulative amount of $\mathrm{CO}_{2}$-C evolved from different plant residues

\begin{tabular}{|c|c|c|c|c|c|c|c|c|c|c|c|c|c|c|c|c|c|c|c|}
\hline \multirow{2}{*}{$\begin{array}{l}\text { 植物 } \\
\text { Plants }\end{array}$} & \multirow{2}{*}{$\begin{array}{c}\text { 残体 } \\
\text { Residues }\end{array}$} & \multicolumn{18}{|c|}{ 培养时间 Incubation days (d) } \\
\hline & & 1 & 2 & 3 & 4 & 6 & 8 & 11 & 14 & 18 & 22 & 26 & 30 & 34 & 38 & 44 & 50 & 56 & 63 \\
\hline \multirow[t]{2}{*}{ 大豆 Glycine max } & 叶片 Leaf & 4.5 & 23.7 & 36.5 & 48.9 & 72.4 & 89.1 & 108.2 & 123.1 & 136.2 & 147.3 & 157.2 & 165.5 & 172.1 & 177.4 & 184.6 & 189.9 & 196.6 & 206.5 \\
\hline & 茎 Stalk & 3.7 & 16.6 & 25.2 & 38.4 & 51.7 & 85.0 & 98.6 & 111.9 & 123.1 & 131.3 & 139.4 & 146.3 & 151.5 & 156.3 & 165.5 & 171.0 & 177.0 & 187.7 \\
\hline 豇豆 Vigna sesquipedalis & 茎 Stalk & 8.8 & 18.6 & 25.1 & 35.4 & 45.8 & 58.2 & 72.3 & 83.9 & 94.7 & 105.5 & 117.4 & 127.8 & 135.7 & 143.7 & 155.9 & 164.1 & 172.6 & 186.7 \\
\hline \multirow[t]{2}{*}{ 甘薯 Dioscorea esculenta } & 叶片 Leaf & 15.2 & 28.0 & 36.1 & 42.9 & 59.0 & 74.0 & 92.4 & 105.1 & 118.2 & 127.9 & 137.0 & 144.5 & 151.9 & 158.8 & 166.7 & 172.3 & 178.9 & 185.1 \\
\hline & 茎 Stalk & 12.4 & 34.6 & 49.4 & 60.3 & 71.3 & 90.0 & 109.4 & 126.3 & 137.9 & 146.8 & 155.6 & 162.8 & 167.9 & 172.5 & 179.9 & 186.1 & 192.5 & 202.6 \\
\hline 丝瓜 Luffa cylindrica & 叶片 Leaf & 23.6 & 41.6 & 57.7 & 72.6 & 96.4 & 113.4 & 130.0 & 141.9 & 154.0 & 165.0 & 173.4 & 181.7 & 186.9 & 191.5 & 197.9 & 202.2 & 206.3 & 210.9 \\
\hline \multirow[t]{3}{*}{ 玉米 Zea mays } & 叶片 Leaf & 13.4 & 24.3 & 31.6 & 41.4 & 51.2 & 63.3 & 80.0 & 94.9 & 107.8 & 119.7 & 130.0 & 138.6 & 145.9 & 153.1 & 163.5 & 169.9 & 178.4 & 191.2 \\
\hline & 茎 Stalk & 1.1 & 27.5 & 45.1 & 50.9 & 56.7 & 65.3 & 78.0 & 90.0 & 100.3 & 110.3 & 120.0 & 129.4 & 137.2 & 145.5 & 157.3 & 166.2 & 175.0 & 187.6 \\
\hline & 根 Root & 4.5 & 11.8 & 16.6 & 24.5 & 32.3 & 39.5 & 51.2 & 63.2 & 73.0 & 83.7 & 93.1 & 102.1 & 109.1 & 115.6 & 125.1 & 132.1 & 138.8 & 150.4 \\
\hline \multirow[t]{2}{*}{ 水稻 Oryza sativa } & 秸秆 Straw & 6.0 & 12.2 & 16.4 & 24.0 & 31.7 & 42.4 & 59.8 & 76.0 & 88.8 & 100.0 & 109.5 & 117.6 & 124.3 & 130.3 & 140.4 & 147.2 & 154.8 & 167.2 \\
\hline & 根 Root & 0.6 & 2.8 & 4.3 & 6.8 & 9.4 & 13.4 & 23.1 & 32.5 & 41.2 & 50.1 & 58.2 & 64.6 & 69.5 & 73.9 & 80.9 & 86.6 & 92.7 & 103.6 \\
\hline 小麦 Triticum aestivum & 根 Root & 1.2 & 3.2 & 4.5 & 7.0 & 9.6 & 14.4 & 24.2 & 34.5 & 45.1 & 55.4 & 63.5 & 72.3 & 78.8 & 85.0 & 94.7 & 100.7 & 109.2 & 122.1 \\
\hline 甘蔗 Saccharum officinarum & 茎 Stalk & 4.4 & 6.8 & 8.4 & 11.7 & 14.9 & 21.4 & 31.1 & 40.7 & 50.2 & 59.3 & 67.6 & 75.4 & 82.3 & 87.8 & 97.1 & 104.1 & 111.4 & 123.2 \\
\hline 高羊茅 Festuca arundinacea & 叶片 Leaf & 9.7 & 21.6 & 29.6 & 48.6 & 67.7 & 91.9 & 120.5 & 139.8 & 153.6 & 166.3 & 177.0 & 187.2 & 196.2 & 208.0 & 218.5 & 225.3 & 235.1 & 244.8 \\
\hline 菖蒲 Acorus calamus & 叶片 Leaf & 4.0 & 9.0 & 12.4 & 21.8 & 31.1 & 42.5 & 60.6 & 76.3 & 89.7 & 102.3 & 114.2 & 124.3 & 134.4 & 142.6 & 154.9 & 163.3 & 171.6 & 183.8 \\
\hline 菱角 Trapa quadrispinosa & 茎 Stalk & 5.5 & 13.5 & 18.8 & 27.5 & 36.2 & 48.3 & 67.5 & 84.5 & 99.0 & 111.0 & 119.1 & 127.4 & 133.9 & 140.4 & 149.3 & 155.2 & 159.1 & 168.5 \\
\hline \multirow[t]{2}{*}{ 海桐 Pittosporum tobira } & 叶片 Leaf & 5.6 & 29.4 & 45.3 & 60.2 & 83.9 & 107.9 & 134.5 & 156.8 & 174.3 & 187.1 & 199.3 & 208.2 & 215.5 & 221.8 & 231.1 & 237.5 & 244.6 & 256.2 \\
\hline & 叶柄 Leafstalk & 9.7 & 25.1 & 35.3 & 46.4 & 57.6 & 67.5 & 80.9 & 92.9 & 103.1 & 112.2 & 121.9 & 131.2 & 138.2 & 144.6 & 154.2 & 161.5 & 169.4 & 182.5 \\
\hline 法国梧桐 Firmiana simplex & 叶片 Leaf & 4.2 & 16.5 & 24.7 & 32.7 & 40.7 & 48.0 & 56.7 & 64.6 & 73.0 & 79.9 & 86.3 & 91.4 & 95.4 & 99.3 & 104.7 & 108.6 & 112.2 & 116.5 \\
\hline
\end{tabular}

表 3 不同植物残体的矿化特征

Table 3 Characteristics of decomposition for various plant residues

\begin{tabular}{|c|c|c|c|c|}
\hline $\begin{array}{c}\text { 植物 } \\
\text { Species }\end{array}$ & $\begin{array}{l}\text { 残体 } \\
\text { Residual }\end{array}$ & $\begin{array}{c}\mathrm{CO}_{2}-\mathrm{C} \text { 矿化率 } \\
\text { Percentage of } \mathrm{CO}_{2} \text {-C released }\end{array}$ & $\begin{array}{l}\text { 一级动力学常数 } \\
\text { First order decay rate }\left(\times 10^{-2} \mathrm{~d}^{-1}\right)\end{array}$ & $R^{2}$ \\
\hline \multirow[t]{2}{*}{ 大豆 Glycine max } & 叶片 Leaf & 47.2 & $1.40(0.10)$ & 0.9035 \\
\hline & 茎 Stalk & 44.9 & $1.27(0.09)$ & 0.8971 \\
\hline 豇豆 Vigna sesquipedalis & 茎 Stalk & 41.2 & $1.00(0.04)$ & 0.9695 \\
\hline \multirow[t]{2}{*}{ 甘薯 Dioscorea esculenta } & 叶片 Leaf & 41.8 & $1.16(0.08)$ & 0.9243 \\
\hline & 茎 Stalk & 50.1 & $1.54(0.12)$ & 0.9034 \\
\hline 丝瓜 Luffa cylindrica & 叶片 Leaf & 59.7 & $2.28(0.21)$ & 0.8966 \\
\hline \multirow[t]{3}{*}{ 玉米 Zea mays } & 叶片 Leaf & 48.5 & $1.31(0.07)$ & 0.9607 \\
\hline & 茎 Stalk & 43.1 & $1.09(0.06)$ & 0.9520 \\
\hline & 根 Root & 37.7 & $0.88(0.03)$ & 0.9742 \\
\hline \multirow[t]{2}{*}{ 水稻 Oryza sativa } & 秸秆 Straw & 43.5 & $1.08(0.04)$ & 0.9656 \\
\hline & 根 Root & 22.5 & $0.44(0.01)$ & 0.9810 \\
\hline 小麦 Triticum aestivum & 根 Root & 27.7 & $0.54(0.01)$ & 0.9904 \\
\hline 甘蔗 Saccharum officinarum & 茎 Stalk & 26.1 & $0.53(0.01)$ & 0.9880 \\
\hline 高羊茅 Festuca arundinacea & 叶片 Leaf & 56.9 & $1.78(0.10)$ & 0.9430 \\
\hline 菖蒲 Acorus calamus & 叶片 Leaf & 40.9 & $0.98(0.03)$ & 0.9757 \\
\hline 菱角 Trapa quadrispinosa & 茎 Stalk & 47.8 & $1.32(0.06)$ & 0.9527 \\
\hline \multirow[t]{2}{*}{ 海桐 Pittospirum tobira } & 叶片 Leaf & 59.3 & $2.03(0.14)$ & 0.9237 \\
\hline & 叶柄 Leafstalk & 42.8 & $1.10(0.07)$ & 0.9460 \\
\hline 法国梧桐 Firmiana simplex & 叶片 Leaf & 24.1 & $0.58(0.04)$ & 0.8956 \\
\hline
\end{tabular}

括号内数值为一级动力学常数的标准误差 Value in the parentheses is the standard error of the first order decay rate

的一级动力学常数、 $\mathrm{CO}_{2}-\mathrm{C}$ 矿化率与相应的全 $\mathrm{N}$ 和 木质素含量作线性相关分析。结果表明，一级动力 学常数分别与全 $\mathrm{N}$ 含量呈显著正相关 $(r=0.4802$, $p<0.05)$ 、与木质素含量和 $\mathrm{N}$ 含量之比呈显著负相 关 $(r=-0.5313, p<0.05)$ 、与木质素含量呈弱负 相关 $(r=-0.4061, p<0.1)$ 。与此相一致, $\mathrm{CO}_{2}-\mathrm{C}$ 矿化率与全 $\mathrm{N}$ 含量及木质素含量的相关系数分别 为 $0.4135(p<0.1)$ 和 $-0.4856(p<0.05)$, 与木质
素含量 : $\mathrm{N}$ 含量之比的相关系数为 $-0.5119(p<$ $0.05)$ 。运用逐步回归方法进一步研究发现, 一级动 力学常数和 $\mathrm{CO}_{2}-\mathrm{C}$ 矿化率均可用全 $\mathrm{N}$ 含量及木质素 含量的线性组合来定量表示：

$$
\begin{aligned}
& k=3.51 \times 10^{-2}+4.61 \times 10^{-4} \mathrm{~N}-1.53 \times 10^{-4} \mathrm{~L} \\
& \left(R^{2}=0.8120, p<0.001, n=19\right) \\
& F_{c}=100+0.974 \mathrm{~N}-0.364 \mathrm{~L} \\
& \left(R^{2}=0.8277, p<0.001, n=19\right)
\end{aligned}
$$


式中, $F_{c}$ 为培养 9 周的 $\mathrm{CO}_{2}$-C 矿化率 $(\%), N$ 和 $L$ 分别为植物残体的初始全 $N$ 含量 $\left(\mathrm{g} \mathrm{kg}^{-1}\right)$ 及木质素 含量 $\left(\mathrm{g} \mathrm{kg}^{-1}\right)$ 。上述结果表明, 植物残体的初始全 $\mathrm{N}$ 含量及木质素含量决定其分解速率。决定系数 $\left(R^{2}\right)$ 的数值说明不同植物残体分解的变异性有 $80 \%$ 以上可由其初始全 $\mathrm{N}$ 含量及木质素含量的差 异得以解释。对于木质素含量类似的植物残体, 含 $\mathrm{N}$ 量高的(如丝瓜叶)比含 $\mathrm{N}$ 量低的(如大豆茎)分解 快 对于含 $\mathrm{N}$ 量类似的植物残体, 木质素含量高的 (如小麦根)比含量低的 (如海桐茎) 分解慢, 含 $\mathrm{N}$ 量 低或木质素含量高的植物残体分解慢 (表 2、表 3)。

\section{2 田间填埋试验}

田间填埋试验结果表明,植物残体间重量损失 的差异十分显著, 其基本趋势与实验室培养结果一 致(图 2), 两者之间的线性相关系数 $\left(R^{2}\right)$ 为 0.8633 $(n=17, p<0.001)$ 。由图 2 所示线性方程的斜率 (1.356 1) 可知, 平均而言,田间填埋 23 周的重量损 失率比实验室培养 9 周的 $\mathrm{CO}_{2}-\mathrm{C}$ 矿化率高 $35 \%$ 左 右。从两者的相关性来看, 在水热状况适宜的条件 下 培养 9 周的 $\mathrm{CO}_{2}$ - $\mathrm{C}$ 矿化量已足以反映不同植物 残体之间的差异。图 2 的结果亦进一步验证了培养 实验的可靠性。

线性相关分析表明 填埋 23 周的重量损失率与 木质素含量呈显著负相关 $(r=-0.5053, p<$ $0.05)$ 、与木质素含量和 $\mathrm{N}$ 含量之比呈弱负相关 $(r=$ $-0.4427, p<0.1)$, 这些结果与培养实验相一致。 将植物残体的重量损失率与初始全 $\mathrm{N}$ 含量、木质素 含量及木质素含量和 $\mathrm{N}$ 含量之比作逐步回归分析, 得到与方程 (3) 类似的结果 :

$$
\begin{aligned}
& F_{w}=150+1.496 N-0.572 L \\
& \left(R^{2}=0.7990, p<0.001, n=17\right)
\end{aligned}
$$

式中， $F_{w}$ 为植物残体的重量损失率 $(\%), N$ 和 $L$ 意义同上。方程 (4) 的结果进一步说明植物残体 在土壤中的分解速率可通过其初始全 $\mathrm{N}$ 含量及木 质素含量进行定量描述。

\section{3 植物残体的易分解比例}

在建立陆地生态系统 $\mathrm{C}$ 循环模型时, 较为困难 的是如何根据植物残体的化学组分来定量表示其易 分解比例。利用 Pinck 等对 11 种一年生植物残体的 分解测定结果,Parton 等 (1987) 在 CENTURY 模型中 用植物残体的初始木质素含量 $(\mathrm{L})$ 与氮含量 $(\mathrm{N})$ 的 比值确定该物料的易分解比例 $\left(F_{m}\right)$ 。其基本假设 是木质素含量低或全 $\mathrm{N}$ 含量高的植物残体具有高 的易分解比例 基本方程为: $F_{m}=0.99-0.018 L / N$ 。
利用该方程，我们计算了各植物残体的易分解组分， 然后分别与培养 9 周的 $\mathrm{CO}_{2}-\mathrm{C}$ 矿化率 $\left(F_{c}\right)$ 及填埋试 验的重量损失率 $\left(F_{w}\right)$ 进行比较。结果表明, 不同植 物残体的 $F_{m}$ 与 $F_{c}$ 和 $F_{w}$ 的变化趋势是一致的, 但有 些则有较大的差异 (图 3)。从数值上看, $F_{m}$ 与 $F_{w}$ 更为接近。由表 1 可以看出, 本研究所选择的 19 种 植物残体代表了 8 类不同的植物，其 $\mathrm{N}$ 含量和木质 素含量均在一个较大的范围内变化。从这个意义上 说，方程(4)具有更普遍的适用性，用其计算的结果 更能代表植物残体的易分解组分。

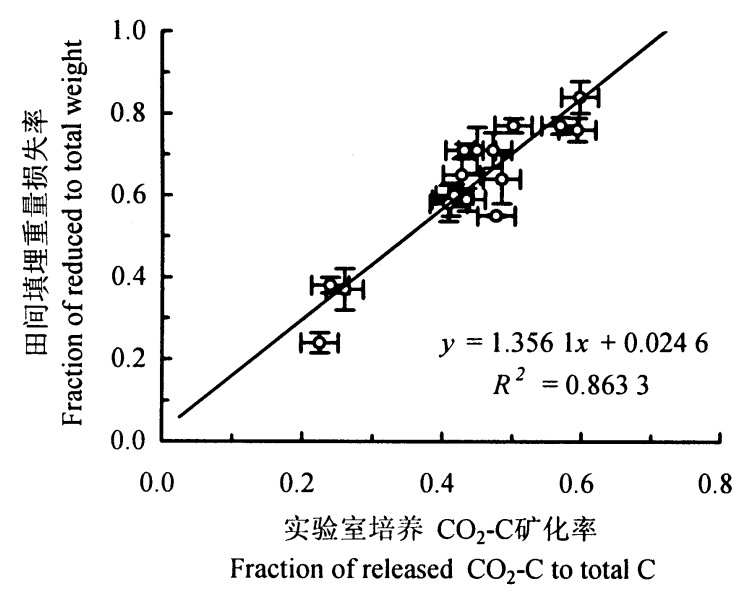

图 2 重量损失率与 $\mathrm{CO}_{2}-\mathrm{C}$ 矿化率比较

Fig.2 Comparison of weight loss from litter bag method with $\mathrm{CO}_{2}-\mathrm{C}$ released from laboratory incubation

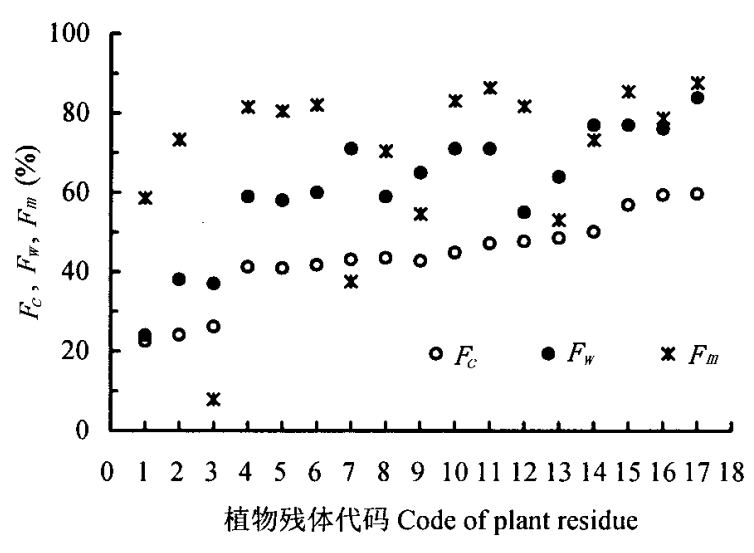

图 3 易分解组分与 $\mathrm{CO}_{2}-\mathrm{C}$ 矿化率及重量损失率比较

Fig. 3 Comparison of $F_{m}$ to $F_{c}$ and $F_{w}$

$F_{c}$ : 实验室培养的 $\mathrm{CO}_{2}-\mathrm{C}$ 矿化率 Percentage of the released $\mathrm{CO}_{2}-\mathrm{C}$ to total $\mathrm{C}$ in laboratory incubation

$F_{w}$ :田间填埋的重量损失率 Percentage of the reduced to total weight in field burying experiment

$F_{m}$ : 易分解比例 Percentage of the labile to total C (Parton et al. , 1987)

\section{3 结 论}

实验室培养实验和田间填埋试验结果表明, 不 
同植物残体的分解速率或重量损失率与其初始全 $\mathrm{N}$ 含量呈正相关、与初始木质素含量或木质素含量 :N 含量之比呈负相关。在水热状况适宜的条件下，培 养 9 周的 $\mathrm{CO}_{2}-\mathrm{C}$ 矿化量已足以反映不同植物残体分 解的差异。用初始含 $\mathrm{N}$ 量和木质素含量两因子的 二元线性模型可以较好地定量描述植物残体分解过 程的一级动力学常数、培养 9 周的 $\mathrm{CO}_{2}-\mathrm{C}$ 矿化率及 填埋 23 周的重量损失率。植物残体的易分解组分 可以通过填埋 23 周的重量损失率进行估计。

\section{参 考 文 献}

Ågren, G. I. , E. Bosatta \& A.H. Magill. 2001 . Combining theory and experiment to understand effects of inorganic nitrogen on litter decomposition. Oecologia, 128:94 $\sim 98$.

Chambers, J. Q., N. Higuchi, J. P. Schimel, L. V. Ferreira \& J. M. Melack. 2000. Decomposition and carbon cycling of dead trees in tropical forests of the central Amazon. Oecologia, 122: $380 \sim 388$.

Eswaran, H., E. van den Berg \& P. Reich. 1993. Organic carbon in soils of the world. Soil Science Society of America Journal, 57: $192 \sim 194$.

Huang, D.M. (黄东迈), P.L. Zhu, (朱培立) \& Z.M. Wang (主志明). 1998. A study and question on the decomposition rate of organic carbon under upland and submerged soil conditions. Acta Pedologica Sinica (土壤学报), 35:482 492. (in Chinese with English abstract)

Huang, Y. (黄耀), S.L. Liu(刘世梁), Q. R. Shen(沈其荣) \& L.G. Zong(宗良纲). 2002. Influence of environmental factors on the decomposition of organic carbon in agricultural soils. Chinese Journal of Applied Ecology (应用生态学报), 13: 709 714. (in Chinese with English abstract)

Kirschbaum, M. U. F. 1995. The temperature dependence of soil organic matter decomposition, and the effect of global warming on soil organic C storage. Soil Biology and Biochemistry, 27:753 760 .

Kwabiah, A. B., R. P. Voroney, C. A. Palm \& N. C. Stoskopf. 1999. Inorganic fertilizer enrichment of soil: effect on decomposition of plant litter under subhumid tropical conditions. Biology and Fertility of Soils, 30:224 231.

Liu, S.L. (刘世梁), Y. Huang (黄耀), Q. R. Shen (沈其 荣), L.G. Zong (宗良纲), D. A. Jiang (蒋定安) \& H. G.
Huang (黄洪光). 2001. Validation and application of a soil organic carbon model. Scientia Agricultura Sinica (中国农业科 学), 34:644 648. (in Chinese)

Lloyd, J. \& J. A. Taylor. 1994. On the temperature dependence of soil respiration. Functional Ecology, 8:315 323 .

Morgan, J.A., D.R. LeCain, A.R. Mosier \& D.G. Milchunas. 2001. Elevated $\mathrm{CO}_{2}$ enhances water relations and productivity and affects gas exchange in $\mathrm{C}_{3}$ and $\mathrm{C}_{4}$ grasses of the Colorado shortgrass steppe. Global Change Biology, 7: 451 466 .

Murayama, S. 1984. Decomposition kinetics of straw saccharides and synthesis of microbial saccharides under field conditions. Journal of Soil Science, 35:231 242 .

Parshotam, A., S. Saggar, P.L. Searle, B.K. Daly, G. P. Sparling \& R. L. Parfitt. 2000. Carbon residence times obtained from labelled ryegrass decomposition in soils under contrasting environmental conditions. Soil Biology and Biochemistry, 32:75 83.

Parton, W.J., D.S. Schimel, C.V. Cole \& D.S. Ojima. 1987. Analysis of factors controlling soil organic matter levels in Great Plains grasslands. Soil Science Society of America Journal, 51: $1173 \sim 1179$.

Reichstein, M. \& F. Bednorz. 2000. Temperature dependence of carbon mineralisation: conclusions from a long-term incubation of subalpine soil samples. Soil Biology and Biochemistry, 32:947 958.

Rutigliano, F. A., A. V. De Santo, B. Berg, A. Alfanil \& A. Fioretto. 1996. Lignin decomposition in decaying leaves of $\mathrm{Fa}$ gus sylvatica L. and needles of Abies Alba mill. Soil Biology and Biochemistry, 28:101 106 .

Schimel, D.S. 1995. Terrestrial ecosystems and the carbon cycle. Global Change Biology, 1:77 91.

Sowerby, A., H. Blum, T.R.G. Gray \& A.S. Ball. 2000. The decomposition of Lolium perenne in soils exposed to elevated $\mathrm{CO}_{2}$ : comparisons of mass loss of litter with soil respiration and soil microbial biomass. Soil Biology and Biochemistry, 32:1359 $\sim 1366$.

Vestergaard, P., R. Rфnn \& S. Christensen. 2001. Reduced particle size of plant material does not stimulate decomposition, but affects the microbivorous microfauna. Soil Biology and Biochemistry, 33: $1805 \sim 1810$.

Weerakoon, W.M., D. M. Olszyk \& D. N. Moss. 1999. Effects of nitrogen nutrition on responses of rice seedlings to carbon dioxide. Agriculture, Ecosystems and Environment, 72: 1 8.

Zhou, L.X. (周立祥). 1995. Study on the dynamics of municipal sewage decomposition and its ecological effect on forest land. Ph. D. dissertation of Nanjing Agricultural University. (in Chinese with English abstract) 